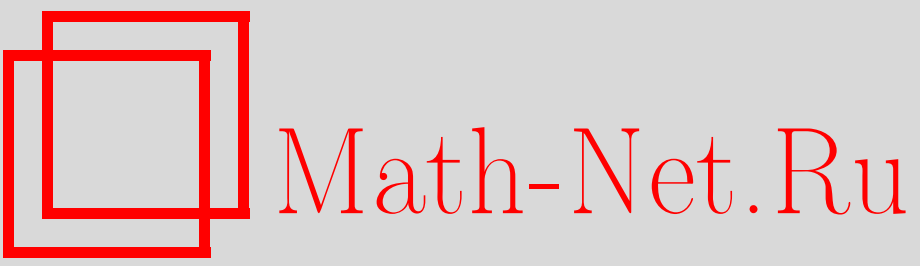

М. И. Штогрин, О строении граней трехмерных многогранников, Изв. РАН. Сер. матем., 2005, том 69, выпуск 4, 205-224

DOI: https://doi.org/10.4213/im653

Использование Общероссийского математического портала Math-Net.Ru подразумевает, что вы прочитали и согласны с пользовательским соглашением

http://www . mathnet.ru/rus/agreement

Параметры загрузки:

IP : 54.157 .27 .8

26 апреля 2023 г., 10:34:38 


\title{
О строении граней трехмерных многогранников
}

\begin{abstract}
В трехмерном евклидовом пространстве рассмотрены двумерные полиэдры, гомеоморфные замкнутым двумерным поверхностям. Подробно исследовано строение произвольной грани полиэдра. В частности, доказана следующая основная теорема: если двумерный полиэдр, расположенный в трехмерном евклидовом пространстве, изометричен поверхности выпуклого трехмерного многогранника, то все грани полиэдра являются выпуклыми многоугольниками.

Библиографиия: 7 наименований.
\end{abstract}

\section{Введение}

Рассмотрим в трехмерном евклидовом пространстве $\mathbb{R}^{3}$ двумерный полиэдр $P$ (см. $[1$, с. 9]). Любая точка $A \in P$ обладает в $P$ окрестностью, являющейся конусом с вершиной $A$ и компактным основанием (см. $[1$, с. 10]). При достаточно малом $\varepsilon>0$, зависяшем от точки $A \in P$, окрестность точки $A$ в $P$ можно задавать в виде пересечения полиэдра $P$ с внутренностью трехмерного шара радиуса $\varepsilon$ с центром в точке $A$. Такая окрестность состоит из всех точек $B \in P \subset \mathbb{R}^{3}$, которые расположены на расстоянии $\|A B\|<\varepsilon$ от точки $A$, где расстояние $\|A B\|$ вычислено в индуцированной метрике, взятой в качестве внутренней метрики полиэдра $P$. При этом расстояние в $\mathbb{R}^{3}$ от точки $A$ до дополнения полиэдра $P$ к окрестности точки $A$ достигается лишь в точках края окрестности. В противном случае следует уменьшить значение $\varepsilon$. Вместо открытых окрестностей иногда удобнее рассматривать замкнутые окрестности [1], которые мы и будем использовать далее.

Понятие метрической $\varepsilon$-окрестности точки $A$ полиэдра $P$ в евклидовом пространстве, введенное в работе [1, с. 10], является основополагающим, относящимся к внешней геометрии полиэдра. В случае двумерного полиэдра $P$ с этим понятием тесно связаны еще три важных понятия: вершины, ребра и грани.

\section{§1. Вершины, ребра, грани}

1.1. Тип окрестности. Далее мы будем полагать, что полиэдр $P$ представляет собой замкнутую двумерную поверхность. Тогда достаточно малая окрестность точки $A$ на поверхности $P \subset \mathbb{R}^{3}$ обладает одновременно следуюшими двумя свойствами:

1) является конусом, так как $P$ - полиэдр (см. $[1$, с. 9$])$;

2 ) гомеоморфна кругу, так как $P$ - замкнутая поверхность.

Работа выполнена при финансовой поддержке Российского фонда фундаментальных исследований (грант № 05-01-00170), программы "Ведущие научные школы" (грант № НШ-2185.2003.1) и программы ОМН РАН “Современные проблемы теоретической математики".

(C) М. И. Штогрин, 2005 
Для точек произвольной замкнутой полиэдральной поверхности $P$ в $\mathbb{R}^{3}$ существуют следующие три (и только три) типа метрических в-окрестностей (см. $[1$, c. 10]):

- mun 2 , когда окрестность точки $A \in P \subset \mathbb{R}^{3}$ представляет собой круг с центром в точке $A$, расположенный в плоскости $\mathbb{R}^{2} \subset \mathbb{R}^{3} ;$ рис. $1, a$;
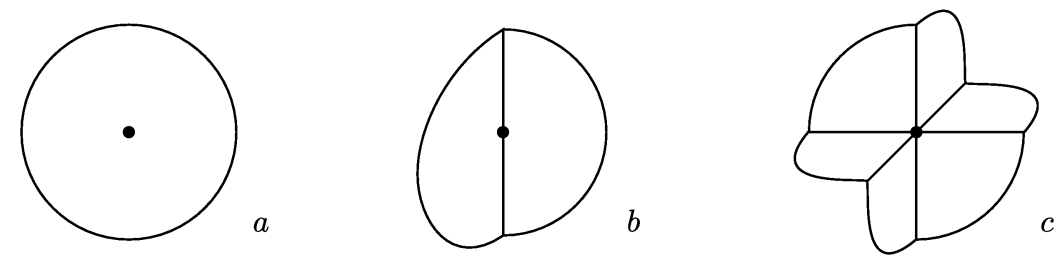

Рис. 1. Три типа метрических $\varepsilon$-окрестностей

- mun 1 , когда окрестность точки $A \in P \subset \mathbb{R}^{3}$ состоит из двух полукругов, расположенных в двух разных ${ }^{1}$ плоскостях и обладаюших одним обшим граничным диаметром (точка $A$ - середина диаметра); см. рис. $1, b$;

- mun 0 , когда окрестность точки $A \in P \subset \mathbb{R}^{3}$ состоит из секторов, все секторы составляют одну циклическую последовательность, соседние секторы последовательности смежны по общему радиусу и расположены в разных плоскостях, несоседние секторы последовательности имеют лиш одну общую точку $A$, число секторов не меньше трех; см. рис. $1, c$.

Для любой точки $A \in P$ при каждом достаточно малом $\varepsilon>0$ метрическая $\varepsilon$-окрестность $N_{\varepsilon}(A, P)$ является конусом (см. [1, с. 10]). Поскольку полиэдр $P$ представляет собой замкнутую двумерную поверхность, то окрестность $N_{\varepsilon}(A, P)$ гомеоморфна кругу. Для каждой точки $B$, расположенной внутри окрестности $N_{\varepsilon}(A, P)$, найдется такое $\delta>0$, при котором имеет место включение $N_{\delta}(B, P) \subset$ $N_{\varepsilon}(A, P)$. Докажем, что окрестность $N_{\delta}(B, P)$ имеет тип 1 или 2 . В самом деле, возьмем образующую $B C$ конуса $N_{\delta}(B, P)$, которая не расположена на прямой, содержащей отрезок $A B$. Все образующие конуса $N_{\varepsilon}(A, P)$, которые пересекают отрезок $B C$, составляют некоторый плоский сектор. Точка $B$ расположена на граничном радиусе этого сектора. Сектору принадлежит только часть окрестности точки $B$ и поэтому вне сектора имеется некоторая образующая $B D$ конуса $N_{\delta}(B, P)$. Все образующие конуса $N_{\varepsilon}(A, P)$, которые пересекают отрезок $B D$, составляют другой плоский сектор. Оба сектора имеют обший радиус. Ему принадлежит отрезок $A B$. Будучи гомеоморфной кругу, окрестность точки $B$ полностью исчерпьвается объединением двух полукругов, каждый из которых расположен в своем секторе. Не умаляя общности, будем считать, что $B C \perp B A$ и $B D \perp B A$. В таком случае двугранный угол между плоскостями обоих секторов измеряется линейным углом $\varphi$ между отрезками $B C$ и $B D, 0<\varphi \leqslant \pi$. В случае $\varphi<\pi$ точка $B$ имеет окрестность типа 1 . В случае $\varphi=\pi$ точка $B$ имеет окрестность типа 2.

Далее, на каждой образующей конуса $N_{\varepsilon}(A, P)$ выберем по точке $B$, расположенной от вершины $A$ на фиксированном расстоянии $\|A B\|=$ const $<\varepsilon$. Получим направляющую $\gamma$. Она представляет собой ограниченное замкнутое множество точек. Докажем, что направляюшая $\gamma$ содержит лишь конечное число точек,

\footnotetext{
${ }^{1}$ Если оба полукруга расположены в одной плоскости, то окрестность точки $A$ имеет тип 2.
} 
окрестности которых имеют тип 1. Предположим, что направляющая $\gamma$ содержит бесконечную последовательность точек, окрестности которьх имеют тип 1. Тогда по лемме Больцано-Вейерштрасса существует бесконечная подпоследовательность, которая сходится к какому-то конечному пределу $B_{0}$. Поскольку направляющая $\gamma$ замкнута, то $B_{0} \in \gamma \subset N_{\varepsilon}(A, P)$. Окрестность точки $B_{0}$ имеет тип 1 или тип 2 (см. выше). В обоих случаях при достаточно малом $\delta>0$ каждая точка пересечения $\left(\gamma \backslash B_{0}\right) \cap N_{\delta}\left(B_{0}, P\right)$ имеет окрестность типа 2. Противоречие. Значит, направляюшая $\gamma$ содержит лишь конечное число точек с окрестностями типа 1. Следовательно, окрестность $N_{\varepsilon}(A, P)$ может иметь лишь один из трех типов: 2,1 или 0.

Кстати сказать, тип 0 допускает разделение на подтипы (по числу сходящихся секторов).

Каждая точка поверхности $P \subset \mathbb{R}^{3}$ имеет свой тип окрестности. При уменьшении радиуса окрестности ее тип не меняется. Для фиксированной точки тип окрестности один из указанных трех.

1.2. Грани. Произвольная замкнутая полиэдральная поверхность $P \subset \mathbb{R}^{3}$ однозначно разбивается на компоненты - линейно связные, относительно открытые, попарно непересекающиеся друг с другом множества, в каждом из которых окрестности всех точек имеют один и тот же тип.

В самом деле, пусть точка $A \in P \subset \mathbb{R}^{3}$ имеет метрическую $\varepsilon$-окрестность типа 0. Тогда все внутренние точки секторов, составляющих данную окрестность, имеют окрестности типа 2. Все внутренние точки радиусов, по которым смежны составляющие окрестность секторы, имеют окрестности типа 1. Одна лишш точка $A$ имеет окрестность типа 0 . Точка $A$ является верииной поверхности $P$.

Пусть, далее, точка $A \in P \subset \mathbb{R}^{3}$ имеет $\varepsilon$-окрестность типа 1 . Тогда все внутренние точки обоих полукругов, составляющих окрестность точки $A$, имеют окрестности типа 2. Все внутренние точки диаметра, по которому пересекаются оба полукруга, обладают окрестностями типа 1 , как и сама точка $A$. Максимальный открытый отрезок (интервал), состоящий из точек поверхности $P$ с окрестностями типа 1 , является ребром поверхности $P$. Оба конца ребра являются вершинами поверхности $P$.

Пусть, наконец, точка $A \in P \subset \mathbb{R}^{3}$ имеет $\varepsilon$-окрестность типа 2 . Тогда все внутренние точки круга, представляющего окрестность точки $A$, обладают окрестностями типа 2. Максимальная линейно связная область, состоящая из точек с окрестностями типа 2 , является гранью поверхности $P$. Любая грань поверхности $P$ ограничена ребрами.

Итак, максимальное линейно связное множество точек на замкнутой полиэдральной поверхности $P \subset \mathbb{R}^{3}$, окрестности которых имеют тип $i$, представляет собой $i$-мерную грань поверхности $P$, где $i=0,1,2$. Таким образом, каждая грань поверхности $P$ представляется нами как компонента связности фазовой области, состоящей из всех точек поверхности $P$ с окрестностями какого-либо фиксированного типа.

Три конкретных примера общего вида грани см. на рис. $2, a-c$.

1.3. Дыры. Рассмотрим произвольную грань замкнутой полиэдральной поверхности $P \subset \mathbb{R}^{3}$. Покажем, что граница грани однозначно распадается на несколько простых замкнутых ломаных (простых реберных циклов по $\bmod 2$ ). Возь- 

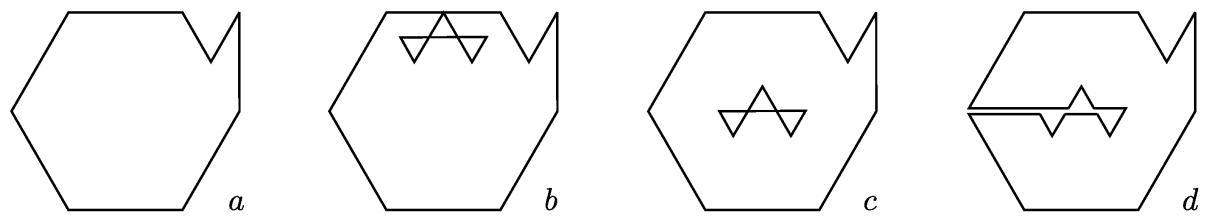

Рис. 2. Три примера граней и разрез неодносвязной грани

мем фиксированную из них, обозначим ее через $z$. По теореме Жордана простая замкнутая ломаная $z$ разбивает плоскость данной грани на две области: внутреннюю и внешнюю. Для ломаной $z$ мы воспользуемся следующим важным заключением из теоремы Жордана: если хотя бы одна точка грани принадлежит ограниченной области, то ни одна точка грани не принадлежст неограниченной области, и, наоборот, если хотя бы одна точка грани принадлежит неограниченной области, то ни одна точка грани не принадлежит ограниченной области.

Ясно, что данная грань целиком содержится внутри одной и только одной ограниченной области, край которой является простым реберным циклом, принадлежашим границе грани. Именно этот цикл мы обозначим z. Наоборот, данная грань находится вне всех остальных ограниченных областей - дыр по отношению к грани, краями которых служат все другие простые реберные циклы, принадлежашие границе данной грани. Эти циклы мы будем обозначать через $z_{j}$. Каждый реберный цикл $z_{j}$ ограничивает свою дыру в данной грани.

Короче говоря, если из плоскости $\mathbb{R}^{2} \subset \mathbb{R}^{3}$, которая содержит данную грань, удалить край грани, то получим в $\mathbb{R}^{2}$ двумерную область, компонентами связности которой являются сама грань, внешность грани, ограниченная циклом $z$, и дыры, ограниченные циклами $z_{j}$. Ясно, что для заданной грани простые реберные циклы $z_{j}$ и $z$ определяются однозначно. Никаких других простых реберных циклов край грани не содержит.

По отношению к любой грани мы будем различать два типа дыр: собственная дыра (дыра в самой грани, рис. 2, c), когда вокруг дыры можно провести замкнутую ломаную, целиком содержашуюся внутри грани (грань неодносвязна, она ограничена не одной уникурсальной ломаной); несобственная дыра (дыра в замыкании грани, рис. $2, b$ ), когда вокруг дыры нельзя провести замкнутую ломаную, содержащуюся внутри рассматриваемой грани, однако вокруг дыры можно провести замкнутую ломаную, целиком содержащуюся в замыкании этой грани.

Если все дыры по отношению к некоторой грани являются несобственными, то эта грань ограничена одной уникурсальной ломаной (см. ниже). При отсутствии дыр по отношению к рассматриваемой грани она ограничена одной простой замкнутой ломаной $z$ (см. рис. $2, a)$, а ее замыкание является односвязным (оно гомеоморфно замкнутому кругу).

1.4. Эйлерова характеристика. Итак, на замкнутой полиэдральной поверхности $P$ в $\mathbb{R}^{3}$ однозначно определяются ее вершины, ребра и грани.

Пусть каждая грань поверхности $P$ ограничена одной уникурсальной ломаной (см. рис. $2, a, b)$. Тогда каждая грань является односвязной (напомним, что любая грань линейно связна по определению) и имеет место формула Эйлера $\chi=v-e+f$, 
где $\chi$ - эйлерова характеристика, $v$ - число вершин, $e$ - число ребер, $f$ - число граней поверхности $P$.

Если хотя бы одну грань поверхности $P$ ограничивает не одна уникурсальная ломаная (см. рис. $2, c)$, то $\chi<v-e+f$, так как внутри каждой неодносвязной грани понадобится дополнительно провести некоторые декоративные ребра, чтобы заменить ее на односвязную грань. (Изображенное на неодносвязной грани декоративное ребро представляет собой два отождествленных ребра односвязной грани; см. рис. $2, c, d$. Односвязная грань расположена по обе стороны от декоративного ребра.)

\section{§ 2. Выпуклые и невыпуклые многоугольники}

2.1. Замкнутая ломаная. Рассмотрим на плоскости $\mathbb{R}^{2}$ простую замкнутую ломаную. Обозначим ее через $z$. Не умаляя общности, будем полагать, что любые два смежных звена ломаной $z$ не лежат на одной и той же прямой. Такая ломаная представляет собой обычный многоугольник, как контур. Контур является замкнутым множеством. Его дополнение к плоскости $\mathbb{R}^{2}$ является открытым. Множество всех точек области $\mathbb{R}^{2} \backslash z$, каждая из которых достигается из какой-либо фиксированной ее точки посредством ломаной, целиком содержащейся в этой области, представляет собой так называемую компоненту связности данной области. Если каждая ломаная, соединяющая какие-либо две точки области $\mathbb{R}^{2} \backslash z$, пересекает ломаную $z$, то эти две точки принадлежат различным компонентам. Как известно, имеет место следуюшая

Теорема (Жордана). Область $\mathbb{R}^{2} \backslash$ z состоит из двух компонент.

Если две простые замкнутые ломаные в евклидовой плоскости $\mathbb{R}^{2}$ находятся в общем положении относительно друг друга, то их пересечение состоит из четного числа точек. В самом деле, одна замкнутая ломаная, обозначим ее через $z$, разбивает плоскость на две области: внутреннюю и внешнюю. Замкнутьй реберный путь вдоль другой ломаной разбивается двумя вершинами $V$ и $W$ на два разомкнутых пути $V . \overrightarrow{.} . W$ и $W . \overrightarrow{.} . V$, следуюшие друг за другом. Точки пересечения пути $V . \overrightarrow{.} . W$ с ломаной $z$ разбивают путь на участки, которые поочередно находятся то во внутренней области, то во внешней области относительно ломаной $z$. Отсюда следует важное для нас заключение: число точек пересечения путu $V . \overrightarrow{.} . W$ с ломаной z (не)четно, если точки $V$ и $W($ не) принадлежат одной и той же области. Четность числа точек пересечения путей $V . \overrightarrow{.} W$ и $W . \overrightarrow{.} V$ с ломаной $z$ одна и та же, а их сумма четна.

2.2. Внутренняя область. Простая замкнутая ломаная $z$, расположенная на плоскости, разбивает ее на две области: ограниченную (конечную, внутреннюю) и неограниченную (бесконечную, внешнюю). Замыкание внутренней области представляет собой простой двумерный многоугольник, обозначим его ${ }^{2}$ через $M$. Плоский двумерный многоугольник $M$ есть объединение ограниченной области int $M$ с ее краем $z=\partial M$.

\footnotetext{
${ }^{2}$ Напомним, что любые два смежных ребра ломаной $z$ не лежат на одной прямой. Следовательно, число звеньев ломаной $z$ совпадает с числом вершин многоугольника $M$ и $\partial M=z$.
} 
ПРЕДЛОЖЕНИЕ 1. Пусть граничный контур простого плоского двумерного многоугольника $M$ не является треугольником. Тогда многоугольник $M$ имеет по меньшей мере одну внутреннюю диагональ.

ДОКАЗАТЕЛЬСТво. Выпуклую оболочку многоугольника $M$ с краем $\partial M=z$ обозначим через $\operatorname{conv}(z)$. Пусть $A$ - какая-то вершина выпуклой оболочки $\operatorname{conv}(z)$, а $B$ и $C$ - конщы звеньев ломаной $z$, примыкающих к вершине $A$. Тогда отрезок $B C$ не является звеном ломаной $z$ (так как ломаная $z$ не является треугольником). Далее, возможны три случая.

1. Ни внутри треугольника $A B C$, ни внутри отрезка $B C$ нет точек ломаной $z$. Поскольку $A, B, C$ - вершины ломаной $z$, то треугольник $A B C$ принадлежит выпуклой оболочке $\operatorname{conv}(z)$. Пусть точка $V$ расположена внутри стороны $B C$. Тогда точка $V$ находится внутри выпуклой оболочки $\operatorname{conv}(z)$. На продолжении отрезка $V A$ через точку $A$ возьмем точку $W$. В таком случае вершина $A$ расположена внутри отрезка $V W$. Поэтому точка $W$ лежит вне выпуклой оболочки $\operatorname{conv}(z)$. Значит, точка $W$ расположена вне многоугольника $M$. А так как отрезок $W V$ пересекает простую замкнутую ломаную $z=C \ldots B \cup B A C$ в одной точке $A$, причем трансверсально, то точка $V$ лежит внутри многоугольника $M$. Следовательно, сторона $B C$ треугольника $A B C$ является внутренней диагональю многоугольника $M$.

2. Внутри треугольника $A B C$ нет точек ломаной $z$, но внутри отрезка $B C$ есть хотя бы одна точка ломаной $z$. Фиксированную внутреннюю точку отрезка $B C$, принадлежащую ломаной $z$, обозначим через $G$ (она существует по предположению). Фиксированную внутреннюю точку отрезка $B C$, не принадлежащую ломаной $z$, обозначим через $H$ (она существует, так как ломаная $z$ не совпадает с граничным контуром треугольника $A B C$ ). Ближайшую к $H$ точку пересечения $G H \cap z$ обозначим через $D$ (на замкнутом конечном множестве минимальное расстояние всегда достигается). Точка $D$ является вершиной ${ }^{3}$ ломаной $z$. Внутри отрезка $A D$ нет точек ломаной $z$ (так как их нет внутри треугольника $A B C$ ). Рассмотрим две точки $V$ и $W$, полагая $V \in \operatorname{int} A D$ и $A \in \operatorname{int} V W$. Точка $W$ лежит вне выпуклой оболочки $\operatorname{conv}(z)$ и вне многоугольника $M$, а точка $V$ лежит внутри многоугольника $M$ и отрезок $A D$ является внутренней диагональю многоугольника $M$.

3. Внутри треугольника $A B C$ есть хотя бы одна точка ломаной z. В таком случае внутри треугольника $A B C$ есть хотя бы одна вершина ломаной $z$. В самом деле, рассмотрим точку ломаной $z$, расположенную внутри треугольника $A B C$. Будем от этой точки постепенно перемешаться вдоль ломаной $z$ в положительном (наугад выбранном) направлении. Поскольку ломаная $z=C \ldots B \cup B A C$ замкнута, то в процессе перемещения по ней (точнее, по разомкнутой ломаной $C \ldots B \subset z$ ) мы должны покинуть внутренность треугольника $A B C$, но сначала должны выйти на его границу. Нельзя выйти на границу сразу либо в точке $A$, либо во внутренней точке отрезка $A B$, либо во внутренней точке отрезка $A C$. Выйти на границу треугольника $A B C$ сразу можно только в точке отрезка $B C$. Фиксируем момент этого первого выхода, который наступает при перемещении вдоль ломаной $z$ в положительном направлении. В момент выхода мы поменяем направление переме-

\footnotetext{
${ }^{3}$ Доказывается от противного. Если $D$ не вершина, то она лежит внутри звена ломаной $z$. Это звено пересекает отрезок $B C$ трансверсально, иначе точка $D$ не была бы ближайшей к $H$. Значит, внутри треугольника $A B C$ имеется некоторая точка ломаной $z$. Противоречие.
} 
щения на отрицательное, и вернемся вдоль ломаной $z$ обратно. Находясь внутри треугольника $A B C$, будем двигаться вдоль ломаной $z$ в отрицательном направлении до тех пор, пока снова не выйдем на границу и опять в точке отрезка $B C$. Так мы получим участок ломаной $z$, расположенный внутри треугольника $A B C$. Концы этого участка принадлежат отрезку $B C$. Конщы участка не могут совпадать, так как ломаная $z=C \ldots B \cup B A C$ не имеет самопересечений и она не может замкнуться в одной точке отрезка $B C$, так как ей принадлежат еще два звена: $A B$ и $A C$. Итак, участок ломаной $z$, расположенный внутри треугольника $A B C$, пересекает отрезок $B C$ в двух разньх точках. Поскольку этот участок имеет точки внутри треугольника $A B C$, он не может принадлежать одному звену ломаной $z$. Следовательно, участок принадлежит по крайней мере двум звеньям. Общий конец двух звеньев участка представляет собой вершину ломаной $z$. Данная вершина расположена внутри треугольника $A B C$.

Внутри треугольника $A B C$ имеется всего лишь конечное число вершин ломаной $z$. Проведем через каждую из них прямую, параллельную отрезку $B C$. Ближайшую к точке $A$ прямую обозначим через $p$. Обозначим точки пересечения прямой $p$ с отрезками $A B$ и $A C$ через $B^{\prime}$ и $C^{\prime}$ соответственно. Внутри треугольника $A B^{\prime} C^{\prime}$ нет точек ломаной $z$ (иначе внутри треугольника $A B^{\prime} C^{\prime}$ нашлась бы вершина ломаной $z$, расположенная на параллельной отрезку $B C$ прямой, более близкой к $A$, чем прямая $p$ ). Обозначим через $D$ вершину ломаной $z$, расположенную внутри отрезка $B^{\prime} C^{\prime}$. Внутри отрезка $A D$ нет точек ломаной $z$. Отрезок $A D$ является внутренней диагональю многоугольника $M$. Предложение доказано.

ПРЕДЛОЖЕНИЕ 2. Произвольный простой плоский двумерный многоугольник $М$ обладает внешнепланарной триангуляцией.

Существование такой триангулящии для выпуклого многоугольника общеизвестно: если число вершин многоугольника равно $n$, то $n-3$ попарно непересекающихся диагоналей разбивают его на $n-2$ треугольника. Доказательство существования триангулящии для невыпуклого многоугольника имеется в [2]. Здесь мы приводим новое доказательство этого факта и вновь подтверждаем, что сумма внутренних углов невыпуклого $n$-угольника равна $(n-2) \pi$.

ДокАЗАТЕЛЬСтво. Если число звеньев простой замкнутой ломаной $z=\partial M$ равно трем, то она ограничивает обычный треугольник. В триангулящию входят один треугольник, три его стороны и три вершины.

Если многоугольник $M$ не является треугольником, то у него имеется хотя бы одна внутренняя диагональ (см. предложение 1). Она разбивает многоугольник на два многоугольника с меньшим числом вершин. Каждый из них, если он не треугольник, опять имеет внутреннюю диагональ и разбивается ею на два многоугольника с меньшим числом вершин и т. д. Через конечное число шагов получим разбиение многоугольника $M$ на треугольники. Это триангуляция. Множество вершин триангуляции совпадает с множеством вершин многоугольника $M$. Множество сторон треугольников состоит из граничных сторон многоугольника $M$ (каждая граничная сторона многоугольника $M$ принадлежит одному треугольнику) и некоторых его внутренних диагоналей (любая такая внутренняя диагональ принадлежит двум треугольникам). Предложение доказано.

Реберный остов данной триангуляции представляет собой внешнепланарный граф (см. [4, с.131]), так как все вершины этого графа принадлежат одной грани 

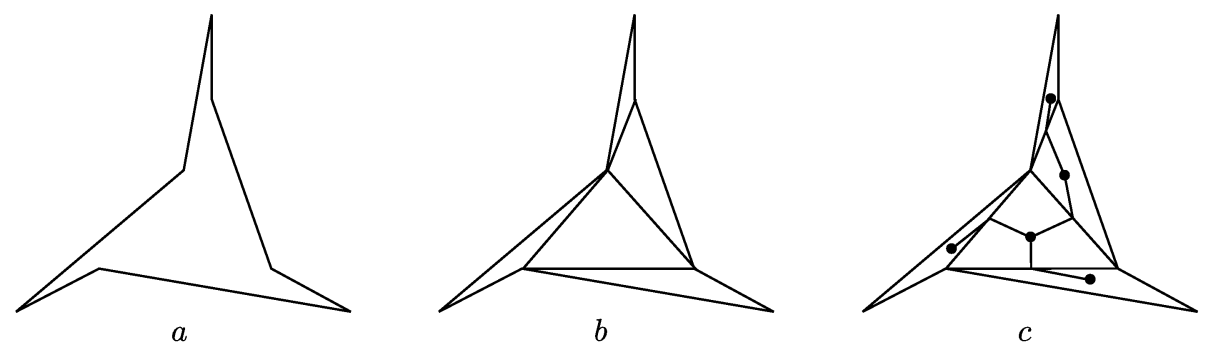

Рис. 3. Многоугольник, внешнепланарная триангуляция и граф смежности

(совпадаюшей с внешностью многоугольника $M$ ). Поэтому и саму триангулящию мы будем называть внешнепланарной, что вполне естественно. Кстати сказать, в некоторых случаях внешнепланарная триангуляция невьпуклого многоугольника является единственной его триангулящией (см., например, рис. $3, a, b)$.

ПРЕДЛОЖЕНИЕ 3. Внешнепланарная триангулячия произвольного многоугольника с п вершинами состоит из $n-2$ треугольников.

ДокАЗАТЕЛЬСтво. Рассмотрим внешнепланарную триангуляцию многоугольника $M$ и построим граф смежности ее треугольников:

- каждому треугольнику заданной внешнепланарной триангуляции многоугольника $M$ поставим в соответствие вершину графа смежности;

- две вершины графа смежности соединим ребром тогда и только тогда, когда соответствующие им два треугольника заданной триангулящии смежны по общей стороне - внутренней диагонали многоугольника $M$.

Граф̆ смежности представим на самой триангулящии, а именно в качестве вершин графа возьмем центры тяжести треугольников (точки пересечения медиан), а каждое ребро графа зададим в виде объединения двух третей медиан треугольников, смежных по стороне (середину общей стороны двух треугольников соединим прямолинейными отрезками с их центрами тяжести - см. рис. $3, c$ ). Пользуясь таким представлением графа смежности, докажем, что он является деревом.

Предположим противное Пусть в графе смежности имеется простой реберный цикл $\gamma$. Весь он содержится внутри триангуляции. По теореме Жордана он разрезает плоскость многоугольника $M$ на две области с краем $\gamma$. Рассмотрим произвольное ребро цикла $\gamma$. Его концы суть центры тяжести двух треугольников, смежных по общей стороне - внутренней диагонали многоугольника $M$. Так как эта диагональ пересекает рассматриваемое ребро цикла $\gamma$ в одной точке, причем трансверсально, то концы диагонали расположены в разных областях относительно $\gamma$. Итак, имеются две вершины многоугольника $M$, расположенные в разных областях относительно $\gamma$. С другой стороны, край многоугольника $M$ является связным и он не пересекается с циклом $\gamma$, так как цикл $\gamma$ расположен строго внутри многоугольника $M$. Получено противоречие. Следовательно, граф смежности является деревом.

Далее, в каждом нетривиальном дереве имеется по меньшей мере две концевые (висячие) вершины ${ }^{4}$. Концевой вершине графа смежности соответствует висячий

\footnotetext{
${ }^{4}$ Исключением служит только тривиальный графф, который состоит лишь из одной вершины. В таком случае исследуемая нами триангуляция состоит всего лишь из одного треугольника.
} 
треугольник триангуляции. Он имеет две граничные стороны, обе являются сторонами многоугольника $M$. Если из внешнепланарной триангуляции удалить висячий треугольник, то триангулящия потеряет одну вершину. Если оставшаяся триангуляция имеет более одного треугольника, то удалим из нее новый висячий треугольник и т. д. На каждом шаге удаления треугольника триангулящия теряет одну вершину. Если исходная внешнепланарная триангулящия имеет $n$ вершин, то через $n-3$ шага от триангулящии останется один треугольник. Отсюда следует, что внешнепланарная триангуляция с $n$ вершинами содержит всего $n-2$ треугольника. Предложение доказано.

СЛЕДСТВИЕ 1. Сумма внутренних углов произвольного многоугольника (как выпуклого, так и невыпуклого) с п вериинами равна $(n-2) \pi$.

ПРЕДЛОЖЕНИЕ 4. Произвольная простая замкнутая ломаная z, расположенная на плоскости $\mathbb{R}^{2}$, имеет по меньшей мере три вериинь, в каждой из которых внутренний угол ломаной z меньше развернутого.

ДоКАЗАТЕЛЬСтво. Предположим противное. Пусть число вершин, в которых внутренние углы ломаной $z$ меньше $\pi$, меньше трех. Тогда среди всех $n$ вершин ломаной $z$ имеются по меньшей мере $n-2$ вершины, в каждой из которых внутренние углы ломаной $z$ не меньше $\pi$. Сумма одних только этих $n-2$ внутренних углов не меньше $(n-2) \pi$. Значит, сумма всех $n$ внутренних углов ломаной $z$ больше $(n-2) \pi$. Противоречие. Следовательно, число внутренних углов ломаной $z$, величина которых меньше $\pi$, не меньше трех. Предложение доказано.

ПРЕДЛОЖЕНИЕ 5. Произвольный простой плоский двумерный многоугольник $М$ можнно изотопно деформировать в один треугольник.

ДоКАЗАТЕЛЬСТво. Согласно доказательству предложения 3 все поочередно удаляемые треугольники внешнепланарной триангулящии многоугольника $M$ обозначим через $\Delta_{1}, \Delta_{2}, \ldots, \Delta_{n-3}$. При каждом $i=1,2, \ldots, n-3$ треугольник $\Delta_{i}$ является висячим в промежуточной триангулящии, состояшей из треугольников $\Delta_{i}, \Delta_{i+1}, \ldots, \Delta_{n-3}, \Delta$. Через $\Delta$ обозначен тот треугольник, которьй остается от триангулящии после завершения всей процедуры удаления висячих треугольников. Если $i=n-3$, то индексу $i+1$ соответствует последний треугольник $\Delta_{n-2}=\Delta$.

Теперь шаг удаления треугольника $\Delta_{i}$ заменим на изотопию. А именно, в промежуточной триангулящии для треугольника $\Delta_{i}$ всегда имеется единственный треугольник $\Delta_{j}$ с индексом $j \in\{i+1, \ldots, n-2\}$, смежный с ним по стороне. Обозначим эту сторону через $S N$ (см. рис. 4$)$.

Недостаюшие вершины треугольников $\Delta_{i}$ и $\Delta_{j}$ обозначим соответственно через $A$ и $B$. Значит, $\Delta_{i}=S N A$ и $\Delta_{j}=S N B$. Середину общей стороны $S N$ обозначим через $C$. На продолжении медианы $C A$ через вершину $A$ возьмем точку $O$, причем столь близкую к $A$, что треугольники $O A S$ и $O A N$ не пересекаются с треугольниками $\Delta_{i+1}, \ldots, \Delta_{n-2}$ (кроме вершин $S$ и $\left.N\right)$. Внутри отрезка $C B$ возьмем некоторую точку $D$.

Каждому фиксированному значению параметра $t$ поставим в соответствие три точки $X, Y, Z$, полагая $X \in \operatorname{int} A C,\|A X\|=\|A C\| \cdot t, Y \in \operatorname{int} C D,\|C Y\|=$ $\|C D\| \cdot t, Z \in \operatorname{int} C A,\|A Z\| \cdot\|C Y\|=\|Z C\| \cdot\|X C\|$, где $0<t<1$. Точка $Z$ 


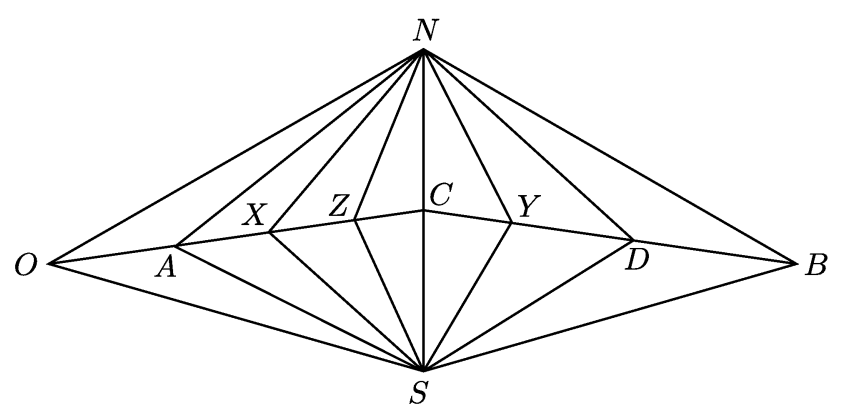

Рис. 4. Четырехугольник с двумя треугольниками триангуляции

однозначно определяется значением параметра $t$, так как она делит отрезок $A C$ в отношении

$$
\lambda=\frac{\|A Z\|}{\|Z C\|}=\frac{\|X C\|}{\|C Y\|}=\frac{\|A C\| \cdot(1-t)}{\|C D\| \cdot t} .
$$

Построим две надстройки над ломаными $O A Z C B$ и $O X C Y B$. Они дают две триангулящии четырехугольника $O S B N$, каждая из которых состоит из восьми треугольников. Теперь воспользуемся тем, что существует кусочно линейное отображение одной триангуляции на другую, а именно

$$
\begin{aligned}
& O A N \rightarrow O X N, \quad A Z N \rightarrow X C N, \quad Z C N \rightarrow C Y N, \quad C B N \rightarrow Y B N, \\
& O A S \rightarrow O X S, \quad A Z S \rightarrow X C S, \quad Z C S \rightarrow C Y S, \quad C B S \rightarrow Y B S .
\end{aligned}
$$

При $t=0$ имеем $X=A, Y=C, Z=C$. В этом случае обе триангулящии четырехугольника $O S B N$ совпадают. Они содержат по шесть треугольников. В пределе при $t \rightarrow 0$ отображение (1) принимает тривиальный вид:

$$
\begin{aligned}
& O A N \rightarrow O A N, \quad A C N \rightarrow A C N, \quad C B N \rightarrow C B N, \\
& O A S \rightarrow O A S, \quad A C S \rightarrow A C S, \quad C B S \rightarrow C B S .
\end{aligned}
$$

При $t=1$ имеем $X=C, Y=D, Z=A$. В данном случае триангулящии четырехугольника $O S B N$ разные. Они опять содержат по шесть треугольников. В пределе при $t \rightarrow 1$ отображение (1) имеет следуюший вид:

$$
\begin{aligned}
& O A N \rightarrow O C N, \quad A C N \rightarrow C D N, \quad C B N \rightarrow D B N, \\
& O A S \rightarrow O C S, \quad A C S \rightarrow C D S, \quad C B S \rightarrow D B S .
\end{aligned}
$$

Итак, при каждом $t$, где $0 \leqslant t \leqslant 1$, построено кусочно линейное отображение четырехугольника $O S B N$ на себя. Оно является тождественным на граничном контуре четырехугольника $O S B N$. Дополняя его тождественным отображением вне четырехугольника $O S B N$, получим изотопное отображение плоскости $\mathbb{R}^{2}$ на себя, при котором объединение треугольников $\Delta_{i} \cup \Delta_{j}$ деформируется в один треугольник $\Delta_{j}$. Применим поочередно изотопию (1) при $i=1,2, \ldots, n-3$. В peзультате получим изотопию многоугольника $M$ на треугольник $\Delta$. Предложение доказано.

ЛЕмма 1. Пусть пересечение простой замкнутой ломаной z, расположенной на плоскости $\mathbb{R}^{2}$, с любой прямой, опорной $\kappa$ ломаной $z$, является связным множеством. Тогда ломаная z является выпуклой. 
ДокАЗАТЕЛЬСТво. Ясно, что выпуклая оболочка $\operatorname{conv}(z)$ является выпуклым многоугольником и все вершины выпуклой оболочки $\operatorname{conv}(z)$ являются вершинами ломаной $z$. Любая прямая, на которой расположена граничная сторона (ребро) выпуклой оболочки $\operatorname{conv}(z)$, является опорной к ней. Обе вершины этого ребра являются вершинами ломаной $z$. Поскольку пересечение ломаной $z$ с любой прямой, опорной к ломаной $z$, по условию данной леммы является связным, то ребро выпуклой оболочки conv $(z)$ вместе со своими конщами целиком принадлежит ломаной $z$. Все ребра выпуклой оболочки $\operatorname{conv}(z)$ составляют простую замкнутую ломаную. Она целиком содержится в $z$. Она не может быть собственной частью ломаной $z$, так как ломаная $z$ сама является простой замкнутой ломаной (при удалении хотя бы одной точки из простой замкнутой ломаной $z$ ее остаток станет разомкнутым). Следовательно, ломаная $z$ полностью совпадает с краем своей выпуклой оболочки $\operatorname{conv}(z)$. Лемма доказана.

СЛЕДСТВИЕ 2. Пусть простая замкнутая ломаная $z$, расположенная $в$ плоскости $\mathbb{R}^{2}$, не является выпуклой. Тогда существует хотя бы одна прямая, опорная $к$ ломаной $z$, которая имеет с ней несвязное пересечение.

ЛЕмма 2. Если простая замкнутая ломаная, расположенная в $\mathbb{R}^{2}$, не является выпуклой, то хотя бы один ее внутренний угол больше $\pi$ (или, что то же самое, хотя бы один ее наружный угол меньше $\pi$ ).

ЗАмЕЧАНИЕ 1. Плоский двумерный угол, образованный смежными звеньями ломаной $z$, называется внутренним, если круг достаточно малого радиуса с центром в вершине вырезает из угла сектор, целиком содержащийся в замыкании внутренней области, ограниченной ломаной $z$ (определение внутреннего угла, данное в [3, с. 749-750], является некорректным).

ЗАмЕЧАНИЕ 2. Плоский двумерный угол, образованный смежными звеньями ломаной $z$, назовем наружнылм, если круг достаточно малого радиуса с центром в вершине вырезает из угла сектор, целиком содержащийся в замыкании внешней области относительно ломаной $z$. Значит, наружный угол - это дополнение внутреннего угла до $2 \pi$ (напомним, что часто применяемый на практике традиционный внешний угол дополняет внутренний угол до $\pi$, а не до $2 \pi)$.

ДоКАЗАТЕЛЬСТво ЛЕммы 2. Пусть простая замкнутая ломаная $z$, расположенная на плоскости $\mathbb{R}^{2}$, невьпукла. Тогда по следствию 2 пересечение ломаной $z$ с некоторой опорной прямой несвязно.

На двух соседних компонентах этого пересечения возьмем две ближайшие друг к другу точки. Обозначим их через $A$ и $B$ (рис. $5, a)$. Точки $A$ и $B$ разбивают простую замкнутую ломаную $z$ на две разомкнутые ломаные - $A L B$ (рис. $5, b$ ) и $A R B$ (рис. $5, c)$. (Здесь в обозначениях разомкнутых ломаных $A L B$ и $A R B$ использованы именные точки $L$ и $R$, которые не являются вершинами ломаной $z$.) Точки $A$ и $B$ лежат на вертикальной прямой. Рассмотрим горизонтальный отрезок $X Y$ (рис. $5, d$ ), пересекающий пустой от точек ломаной $z$ отрезок $A B$ во внутренней точке $U$.

Далее, пусть точка $X$ расположена левее левой вертикальной опорной к ломаной $z$, а точка $Y$ расположена правее правой вертикальной опорной к ломаной $z$ (см. рис. $5, d)$. Тогда $X Y \cap z \neq \varnothing$. 

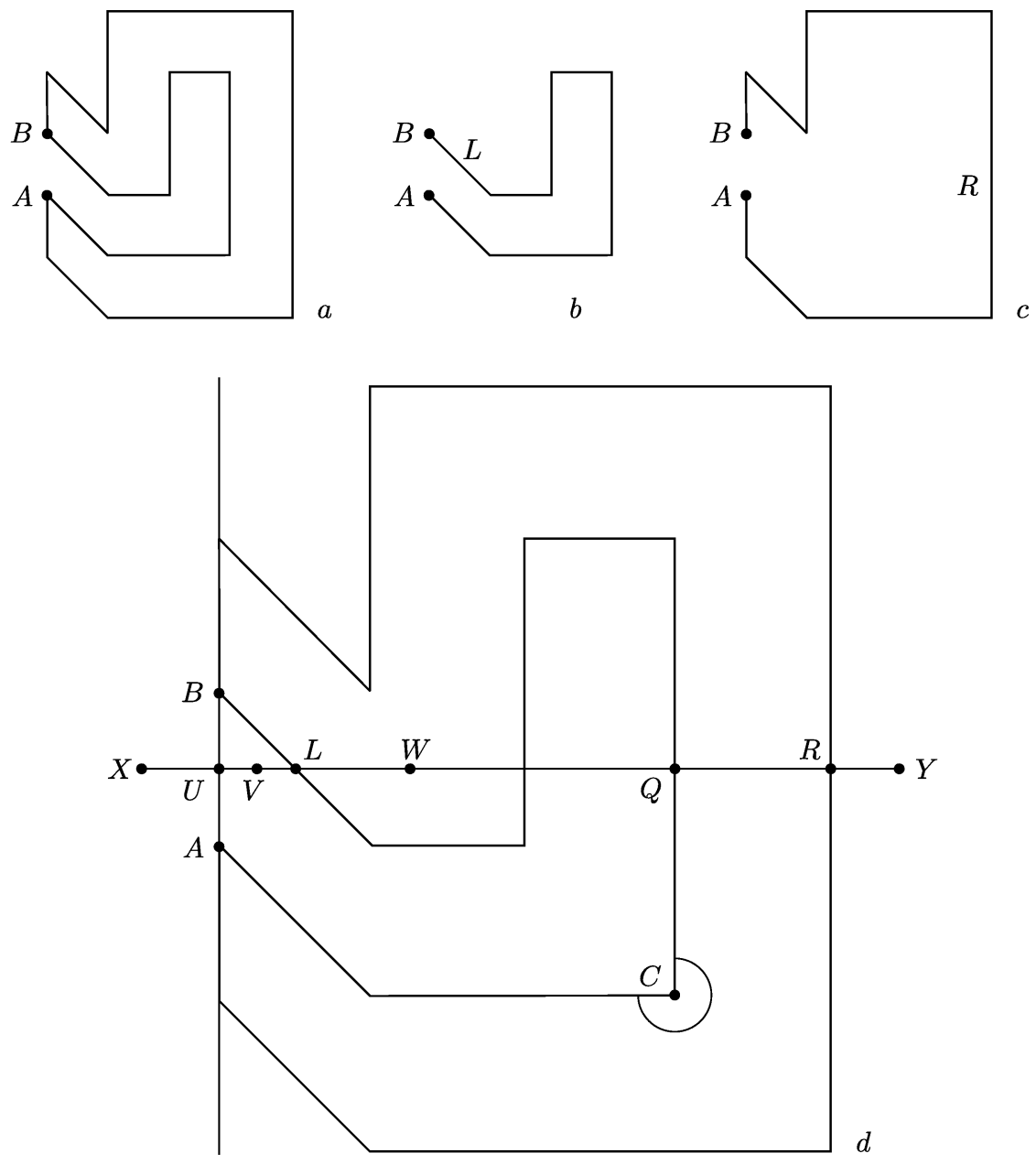

Рис. 5. Невыпуклая ломаная имеет внутренний угол больше $\pi$

Не умаляя общности, будем полагать, что отрезок $X Y$ находится в общем положении по отношению к ломаной $z$, т. е. он не содержит вершин ломаной $z$. Отрезок $X Y$ пересекает лишь ребра ломаной $z$, и каждое локальное пересечение является трансверсальным. На отрезке $X Y$ имеются следующие вспомогательные точки:

$L$ - крайняя левая точка пересечения отрезка $X Y$ с ломаной $z$;

$Q$-крайняя правая точка пересечения отрезка $X Y$ с разомкнутой ломаной $A L B$ (ниже показано, что точка $Q$ не совпадает с точкой $R$ );

$R$ - крайняя правая точка пересечения отрезка $X Y$ с ломаной $z$;

$U$ - точка пересечения отрезка $X Y$ с отрезком $A B$;

$V$ - произвольная внутренняя точка отрезка $U L$;

$W$ - такая точка отрезка $X Y$, что $V W \cap z=L$.

Отрезок $X V$ расположен вне области, ограниченной простой замкнутой ломаной $z$. Точка $W$ расположена внутри области, ограниченной ломаной $z$. Поскольку отрезок $X W$ пересекает ломаную $z$ в одной точке $L$, а точка $X$ лежит вне области, ограниченной ломаной $z$, то отрезок $X V$ лежит вне этой области. Так как 
отрезок $X V$ пересекает простую замкнутую ломаную $A L B \cup B A$ в одной точке $U$, а точка $X$ лежит вне области, ограниченной ломаной $A L B \cup B A$, то точка $V$ лежит внутри этой области. Поскольку отрезок $V W$ пересекает простую замкнутую ломаную $A L B \cup B A$ в одной точке $L$, а точка $V$ лежит внутри области, ограниченной ломаной $A L B \cup B A$, то точка $W$ лежит вне этой области. Так как отрезок $X W$ пересекает простую замкнутую ломаную $A R B \cup B A$ в одной точке $U$, а точка $X$ лежит вне области, ограниченной ломаной $A R B \cup B A$, то отрезок $V W$ лежит внутри этой области. Следовательно, точка $L$, а вместе с ней и вся разомкнутая ломаная $A L B$, в том числе и ее точка ${ }^{5} Q$, лежат внутри этой области. Поскольку $Q$ лежит внутри, а $Y$ - вне области, ограниченной ломаной $A R B \cup B A$, то отрезок $Q Y$ пересекает замкнутую ломаную $A R B \cup B A$ по меньшей мере в одной точке. Крайняя правая точка этого пересечения ранее уже была обозначена нами через $R$. Ясно, что $R \neq Q$, так как $Q$ лежит внутри области, а $R$ - на ее границе.

Возьмем разомкнутую ломаную $A L B$. Замкнем ее отрезком $B A$. Получим простую замкнутую ломаную $A L B \cup B A$. Используя предложение 4 , получим, что замкнутая ломаная $A L B \cup B A$ имеет по крайней мере три вершины, в которых ее внутренние углы меньше $\pi$. Двумя из таких вершин могут быть вершины $A$ и $B$. Следовательно, существует еще хотя бы одна вершина разомкнутой ломаной $A L B$ (обозначим эту вершину через $C$ ), в которой внутренний угол замкнутой ломаной $A L B \cup B A$ меньше $\pi$. Ясно, что в вершине $C$ внутренний угол замкнутой ломаной $A L B \cup B A$ является наружным углом для области, которая ограничена ломаной $z$. Следовательно, в вершине $C$ внутренний угол простой замкнутой ломаной $z$ больше $\pi$. Лемма доказана.

СлЕДСТВИЕ 3. Если все внутренние угль простой замкнутой ломаной $z$, расположенной на плоскости $\mathbb{R}^{2}$, не превышают $\pi$, то плоский двумерный многоугольник, ограниченный ломаной $z$, является выпуклым.

\section{§3. Невыпуклые многогранники}

Продолжим исследование замкнутой полиэдральной поверхности $P$ в пространстве $\mathbb{R}^{3}$. Рассмотрим ее фиксированную грань. Она расположена в некоторой плоскости $\mathbb{R}^{2} \subset \mathbb{R}^{3}$. В этой плоскости, вообще говоря, могут находиться разные грани данной поверхности $P$. Две точки с окрестностями типа 2 (рис. $1, a)$, расположенные в одной и той же плоскости, принадлежат одной и той же грани тогда и только тогда, когда их можно соединить друг с другом посредством ломаной, все точки которой имеют окрестности типа 2 . Произвольная грань поверхности $P$ представляет собой открытую связную плоскую двумерную область.

Гранища грани состоит из ребер поверхности и распадается на несколько простых реберных циклов. Ранее мы обозначили их через $z$ и $z_{j}$. Грань не содержит никаких других простых реберных циклов, кроме указанных циклов $z_{j}$ и $z$. Каждый цикл $z_{j}$ ограничивает дыру в грани. Цикл $z$ находится в особом положении: он ограничивает грань, взятую вместе со всеми своими дырами.

(Эта грань является гранью некоторой полиэдральной сферы. В самом деле, для каждой дыры грани возьмем ее внешнепланарную триангуляцию и над каждым контуром треугольника построим конус-воронку под плоскостью грани. Над

\footnotetext{
${ }^{5}$ Не исключено, что $Q=L$, однако это не меняет сути рассуждений.
} 
плоскостью грани построим конус-крышу с основанием $z$. Грань и все эти конусы вместе составляют полиэдральную сферу с данной гранью. Значит, устройство грани не определяется родом поверхности.)

Каждая дыра (открытая, линейно связная) ограничена простой замкнутой ломаной. Поэтому дыра является односвязной и ее замыкание также остается односвязным множествами. Внутри дыры не содержится никаких точек данной грани. Возможно, что внутри дыры содержатся другие грани поверхности $P$ (или ее ребра, или вершины). Этот вопрос мы здесь не рассматриваем, а изучаем устройство только одной наугад выбранной грани.

Если все дыры по отношению к некоторой грани являются несобственными, то эта грань ограничена одной уникурсальной ломаной (см. ниже). Грань без собственных дыр является открытой односвязной областью, и по отношению к ней несобственная дыра представляется нами, образно говоря, еще как “залив", однако замыкание грани без собственных дыр при наличии хотя бы одной несобственной дыры неодносвязно, а по отношению к нему несобственная дыра представляется нами уже как "озеро". При отсутствии всяких дыр по отношению к рассматриваемой грани эта грань ограничена простой замкнутой ломаной (см. рис. $2, a)$, и ее замыкание односвязно (оно гомеоморфно замкнутому кругу).

При исследовании собственных и несобственных дыр могло создаться впечатление, будто любые две дыры данной грани находятся далеко друг от друга. Но это не всегда так. Две различные дыры как открытые области не имеют общих внутренних точек. Однако их замыкания могут иметь непустые пересечения. Докажем, что пересечение замыканий двух дыр состоит из не более чем одной точки. Пусть, от противного, пересечение замыканий двух дыр имеет две точки $A$ и $B$. Обе точки лежат на границе обеих дыр. Построим две простые разомкнутые ломаные $A x B$ и $B Y A$, каждая из которых ${ }^{6}$ находится внутри своей дыры. Тогда простая замкнутая ломаная $A x B \cup B Y A$ разбивает плоскость грани на две области. Край каждой дыры разбивается на две части, расположенные в разных областях. К обеим частям примыкает грань поверхности $P$ с двумя этими дырами. Получили противоречие с тем, что грань как линейно связная область, не пересекающаяся с ломаной $A x B \cup B Y A$, не может иметь свои точки одновременно в этих двух областях. Значит, любые два простых реберных цикла, являющихся краями двух дыр, могут пересекаться между собой не более чем в одной точке.

Заметим, что дыра к дыре может примкнуть только по вершине поверхности $P$, так как вдоль всего ребра поверхности $P$, вошедшего в край дыры, примыкает грань.

Точно так же доказывается, что произвольный цикл $z_{j}$ (край дыры) и цикл $z$ (край внешней грани) пересекаются не более чем в одной вершине.

Более обшо, любая последовательность из циклов $z_{j}$ и $z$, в которой любые два соседних цикла пересекаются в одной обшей вершине, являющейся своей для каждой пересекающейся пары (т. е. разные пары соседних циклов пересекаются в разных вершинах), не является циклической. Доказывается точно так же, как это сделано, в частности, для последовательности из двух циклов (см. выше).

Итак, в реберном остове грани каждая последовательность простых циклов, в которой любые два соседних цикла пересекаются, причем разные пары соседних

\footnotetext{
${ }^{6}$ Обозначения $X$ и $Y$ меньшим кеглем следует воспринимать как именные отметки на двух этих ломаных.
} 
циклов пересекаются в разных вершинах, обладает следующим свойством: она является ациклической.

Рассмотрим объединение всех простых циклов $z_{j}$, кроме $z$. Объединение $\cup z_{j}$ однозначно распадается на компоненты связности. Любая компонента является краем для некоторого объединения дыр, и мы будем говорить, что все эти дыры вместе с краем составляют так назьваемый каскад ${ }^{7}$ дыр грани. Ясно, что если одна дыра каскада является (не)собственной, то и все остальные дыры каскада являются (не)собственными. Каждый каскад, состоящий из собственных дыр, не пересекается с циклом $z$. Любой каскад, состоящий из несобственных дыр, пересекается с циклом $z$, причем только в одной вершине.

Рассмотрим каскад дыр, в котором каждая дыра является собственной. Рассмотрим ту вершину каскада, к которой примыкают по меньшей мере две дыры; сходяшиеся в вершине простые циклы составляют букет окружностей. Возьмем в этой вершине фиксированный угол грани. Он образован двумя сторонами грани. По одной стороне к грани примыкает одна дыра, по другой стороне - другая дыра. Их граничные реберные циклы пересекаются в данной вершине. Слегка "спилим" выбранный угол в окрестности вершины. Тогда обе дыры объединятся в одну дыру. Однако сформулированное выше свойство ацикличности в новом каскаде дыр сохранится. Повторим этот процесс несколько раз. В итоге все дыры каскада объединятся в одну дыру. Полученная дыра ограничена одним простым замкнутым реберным путем. Восстановив все "спиленные" вершины, получим замкнутый самопересекающийся реберный путь. Он проходит по всем граничным ребрам каскада одним росчерком пера.

Точно так же показывается, что все имеющиеся каскады с несобственными дырами вместе с внешностью грани также ограничены одной уникурсальной ломаной.

Подведем краткий итог доказанного. Назовем два ребра грани смежнылми, если они являются сторонами одного и того же ее угла. Каждое ребро грани имеет два (и только два) смежных с ним ребра. Теперь доказанное выше мы переформулируем в виде следующего утверждения.

УТВЕРЖДЕНИЕ 1. Все ребра каждой компоненты связности границы грани составляют одну ииклическую последовательность, в которой любые два соседних ребра являются смежными.

Рассмотрим произвольный каскад, который содержит не менее двух дыр. Реберный остов каскада представляет собой граф, блоками которого служат циклы $z_{j}$, являюшиеся краями вошедших в каскад дыр. Для него построим граф блоков и точек сочленения (см. [4, с.53]). Напомним, что край любой дыры каскада представляет собой простой цикл. Это блок. Иных блоков на краю каскада нет. Для реберного остова каскада граф блоков и точек сочленения является деревом. Дерево имеет по меньшей мере две коншевые вершины. Каждая концевая вершина соответствует висячему блоку. Любой висячий блок представляет собой простой реберный цикл $z_{j}$, который имеет всего лишь одну точку сочленения (значит, висячая дыра имеет одну точку сочленения).

\footnotetext{
${ }^{7}$ Все дыры грани являются открытыми областями. Дыры попарно не пересекаются между собой. Однако замыкания дыр могут пересекаться друг с другом. Введенный нами каскад представляет собой компоненту связности в объединении замыканий всех дыр данной грани.
} 
Граф̆ блоков и точек сочленения представим на каскаде. А именно, внутри каждой дыры каскада выберем по одной точке, каждую из которых возьмем в качестве вершины, соответствуюшей блоку. В качестве вершин, соответствующих точкам сочленения циклов каскада, возьмем сами точки сочленения. Далее, внутри каждой дыры проведем попарно непересекаюшиеся ребра (дуги или ломаные), которые соединяют выбранную в ней вершину со всеми точками сочленения, принадлежашими ее краю. Граф блоков и точек сочленения построен. Он является деформационным ретрактом каскада. Это дерево. Значит, каскад является односвязным.

ЛЕмма 3. Пусть грань замкнутой полиәдральной поверхности $Р$ не является выпуклой. Тогда грань имеет хотя бы один угол больше $\pi$.

ДоказАтельство. Пусть грань поверхности $P$ невыпукла. Тогда все дальнейшие рассуждения можно разделить на следующие три случая.

1. Грань неодносвязна. Грань имеет хотя бы одну собственную дыру. Если замыкание этой дыры не пересекается с замыканиями всех остальных дыр, то каждый угол дыры дополняет угол грани до $2 \pi$. В силу предложения 4 дыра имеет угол меньше $\pi$. Значит, грань имеет угол больше $\pi$. Если же замыкание дыры пересекается с замыканием другой дыры, то рассмотрим содержащий ее каскад дыр. Для реберного остова каскада построим граф блоков и точек сочленения и воспользуемся тем, что каскад дыр имеет висячую дыру (напомним, что только одна вершина висячей дыры является точкой сочленения). В силу предложения 4 висячая дыра имеет как минимум три угла меньше $\pi$. По крайней мере два из них заведомо являются дополнениями внутренних углов грани до $2 \pi$ (вершина каждого из них не является точкой сочленения).

2. Грань односвязна, но замыканиеграни неодносвязно. Собственных дыр у грани нет. Имеется несобственная дыра. Если замыкание дыры не пересекается с замыканиями всех остальных дыр, то все углы дыры, за исключением одного угла, вершина которого принадлежит ломаной $z$, дополняют угол грани до $2 \pi$. В силу предложения 4 дыра имеет три угла меньше $\pi$. Значит, грань заведомо имеет два угла больше $\pi$. Если же замыкание дыры пересекается с замыканием какой-то дыры, то рассмотрим содержаший ее каскад дыр, а в нем висячую дыру. Одна вершина висячей дыры является точкой сочленения. Может оказаться, что другая вершина дыры принадлежит циклу $z$ (напомним, что циклы $z_{j}$ и $z$ пересекаются не более чем в одной точке). В силу предложения 4 висячая дыра имеет три угла меньше $\pi$. Один из трех этих углов заведомо является дополнением внутреннего угла грани до $2 \pi$.

3. Грань односвязна и замыкание грани односвязно. В таком случае как собственных, так и несобственных дыр у грани нет. Грань ограничена лишш одним простым реберным циклом $z$, а все простые циклы $z_{j}$ реально отсутствуют. Поскольку по условию данной леммы грань не является выпуклой, то согласно лемме 2 по меньшей мере один внутренний угол простой замкнутой ломаной $z$ больше $\pi$.

Во всех трех случаях грань имеет угол больше $\pi$. Лемма доказана.

СЛЕДСТВИЕ 4. Если все плоские угль грани замкнутой полиэдральной поверхности $Р$ не превышают $\pi$, то эта грань является выпуклой.

Такая грань, во-первых, не имеет собственных дыр, во-вторых, не имеет несобственных дыр, в-третьих, ограничена простой замкнутой ломаной, углы которой 
не превышают $\pi$ (если бы эта ломаная не была выпуклой, то по лемме 2 она имела бы внутренний угол, величина которого больше $\pi$, что противоречило бы сделанному в формулировке следствия 4 предположению). Короче говоря, имеющаяся локальная выпуклость влечет глобальную выпуклость.

\section{§4. Положительность кривизны и выпуклость граней}

Продолжим исследование полиэдральной поверхности. Возьмем фиксированную вершину $A$ поверхности $P$. В вершине $A$ сходятся не менее чем три плоских угла поверхности. Все плоские углы, сходяшиеся в вершине $A$, составляют одну циклическую последовательность ${ }^{8}$. В данной циклической последовательности любые два смежных угла расположены в разных плоскостях. Все углы заданной циклической последовательности будем обозначать через $\alpha_{1}, \alpha_{2}, \ldots, \alpha_{k}$, где $3 \leqslant k<\infty$. В этих обозначениях кривизна поверхности $P$ в вершине $A$ вычисляется по формуле

$$
\omega=2 \pi-\sum_{i=1}^{k} \alpha_{i} .
$$

ЛЕмМа 4. Если в вериине поверхности Р имеется плоский угол, величина которого не меньше $\pi$, то кривизна в вершине отрицательна.

ДокАЗАТЕльство. Не умаляя общности, будем полагать, что $\alpha_{1} \geqslant \pi$. Докажем, что имеет место следующее неравенство:

$$
\sum_{i=2}^{k} \alpha_{i}>2 \pi-\alpha_{1}
$$

Доказательство неравенства (5) мы разделим на следующие два случая.

1. Пусть имеет место строгое неравенство $\alpha_{1}>\pi$. Тогда из неравенств $\pi<$ $\alpha_{1}<2 \pi$ следует $0<2 \pi-\alpha_{1}<\pi$. На единичной сфере вокруг вершины та дуга большой окружности, которая стягивает угол $\beta=2 \pi-\alpha_{1}$, является кратчайшей между теми двумя точками на сфере, по которым она пересекается с граничными лучами плоского угла $\alpha_{1}$. Сферическая ломаная, составленная из дуг $\alpha_{2}, \ldots, \alpha_{k}$, взятых из циклической последовательности $\alpha_{1}, \alpha_{2}, \ldots, \alpha_{k}$, является более длинной, чем замыкающая дуга $\beta=2 \pi-\alpha_{1}$. Неравенство (5) доказано.

2. Пусть выполнено равенство ${ }^{9} \alpha_{1}=\pi$. Тогда граничные лучи плоского угла $\alpha_{1}$ лежат на одной прямой и пересекают единичную сферу вокруг его вершины в двух полюсах. Любая кратчайшая линия между полюсами на сфере, как меридиан, равна $\pi$. Сферическая ломаная, составленная из дуг $\alpha_{2}, \ldots, \alpha_{k}$ циклической последовательности $\alpha_{1}, \alpha_{2}, \ldots, \alpha_{k}$, больше длины замыкаюшего меридиана. Поскольку длина меридиана равна $\pi=2 \pi-\alpha_{1}$, то вновь получаем неравенство (5).

Итак, из неравенства $\alpha_{1} \geqslant \pi$ вытекает неравенство (5). В силу формулы (4) из (5) следует неравенство $\omega<0$. Лемма доказана.

\footnotetext{
${ }^{8}$ В циклической последовательности содержится лишь конечное число плоских углов.

9 Здесь настоящая вершина полиэдра является декоративной вершиной грани с углом $\alpha_{1}=\pi$.
} 
Если два полюса на сфере соединены простой линией, состоящей только из двух дуг больших окружностей, то обе дуги вместе составляют одну дугу - цельй меридиан. Поэтому в случае $\alpha_{1}=\pi$ вьполнение неравенства $k \geqslant 3$ влечет вьполнение более сильного неравенства $k \geqslant 4$.

СлЕДСТВИЕ 5. Если в какой-либо вершине поверхности Р кривизна неотрицательна, то каждый плоский угол в данной вершине меньше $\pi$.

Отсюда и из следствия 4 вытекает основная теорема [5].

ТЕОРемА. Пусть для замкнутой полиэдральной поверхности $P$, расположенной в трехмерном евклидовом пространстве $\mathbb{R}^{3}$, кривизна в каждой вериине является неотрицательной. Тогда все грани данной поверхности являются выпукльми многоугольниками.

Сформулированное здесь условие является достаточным, но не является необходимым.

При условиях теоремы имеются всего только две возможности рода поверхности. А именно, если в каждой вершине кривизна равна нулю, то поверхность гомеоморфна тору. Если хотя бы в одной вершине кривизна положительна ${ }^{10}$, то поверхность гомеоморфна сфере. В самом деле, так как поверхность $P$ вложена в пространство $\mathbb{R}^{3}$, то она является ориентируемой. Далее, по условиям теоремы в каждой вершине поверхности $P$ локальная кривизна неотрицательна, т. е. $\omega \geqslant 0$. Поэтому полная кривизна поверхности $\Omega=\sum \omega$ также неотрицательна, т.е. $\Omega \geqslant 0$. Как известно, $\Omega=2 \pi \chi$, где $\chi=2(1-g)$, а $g-$ род поверхности. Следовательно, $g \leqslant 1$.

Для абстрактных разверток неотрицательной кривизны, гомеоморфных двумерной сфере $\mathbb{S}^{2}$, А. Д. Александров [2] доказал теорему существования реализации развертки в виде поверхности выпуклого многогранника в $\mathbb{R}^{3}$ и теорему единственности реализации в классе всех выпуклых многогранников. С. П. Оловянишников [6] доказал единственность реализации в классе всех выпуклых тел. Обе теоремы единственности являются обобшениями следуюшей теоремы Коши [7]: если два замкнутых выпуклых многогранника состоят из попарно конгруэнтных граней, склеенных в одном и том же порядке, то и сами эти многогранники конгруэнтны.

Для любой абстрактной развертки неотрицательной кривизны, гомеоморфной cфере $\mathbb{S}^{2}$, помимо единственного выпуклого вложения в $\mathbb{R}^{3}$ сушествуют сколь угодно много невыпуклых полиэдральных вложений и в каждом из них все грани являются выпуклыми многоугольниками.

У выпуклого многогранника окрестность вершины с плоским краем можно заменить на ее образ при зеркальном отражении от плоскости края. В качестве нормали к плоскости края следует взять вектор, соединяюший вершину многогранника с любой внутренней точкой ее сферического изображения. При этом расстояние от плоскости до вершины должно быть достаточно малым.

В формулировке теоремы вложение можно заменить на погружение или на более общее изометрическое отображение, которое не является погружением, однако после удаления из поверхности $P$ конечного числа точек отображение станет погружением. При этом добавятся еще две неориентируемые поверхности: бутылка Кляйна и проективная плоскость.

\footnotetext{
${ }^{10}$ Тогда общее число вершин с положительной кривизной на самом деле не меньше трех.
} 
ЗАмечАниЕ 3 . Для замкнутой поверхности $P$ произвольного рода, ориентируемой или неориентируемой, кусочно линейно погруженной в трехмерное евклидово пространство $\mathbb{R}^{3}$, необходимые и достаточные условия вьпуклости граней состоят в том, чтобы все плоские углы каждой грани не превышали $\pi$. В случае неотрищательности кривизны в каждой вершине эти условия будут выполнены при любом кусочно линейном изометрическом погружении поверхности $P$ в $\mathbb{R}^{3}$.

Примеры кусочно линейных изометрических отображений в $\mathbb{R}^{3}$ для поверхностей постоянной нулевой кривизны даны на рис. $6, a$ и $7, a$.
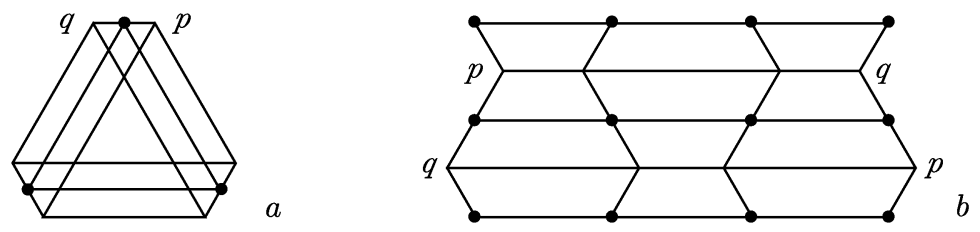

Рис. 6. Проекция бутылки Кляйна и ее развертка

На рис. $6, a$ изображена проекция бутылки Кляйна на зеркальную плоскость симметрии, перпендикулярную тройной оси симметрии. Поверхность имеет 6 особых точек, из них 3 под и 3 над плоскостью проекции (последние на рисунке отмечены жирно). Окрестность особой точки представляет собой зонтик Уитни. Поверхность имеет 12 граней - 6 больших и 6 малых трапеций. Развертка поверхности представлена на рис. $6, b$.

В качестве порядка оси симметрии можно взять произвольное нечетное число $2 n+1$ при каждом натуральном $n$. Таким образом, получается бесконечная серия примеров кусочно линейных изометрических отображений бутылки Кляйна постоянной нулевой кривизны в трехмерное евклидово пространство $\mathbb{R}^{3}$. На рис. $6, a$ поверхность из данной серии имеет наименьший порядок оси симметрии, он равен $2 n+1=3$.
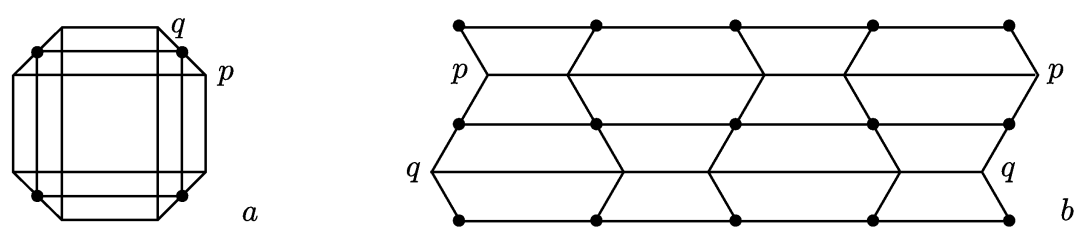

Рис. 7. Проекция тора и его развертка

На рис. $7, a$ изображена проекция тора на зеркальную плоскость симметрии, перпендикулярную четверной оси симметрии. Поверхность имеет 8 особых точек, из них 4 под и 4 над плоскостью проекции. Окрестность каждой особой точки также является зонтиком Уитни. Поверхность имеет 16 граней -8 больших и 8 малых трапеций. Развертка поверхности дана на рис. $7, b$.

В качестве порядка оси симметрии можно взять произвольное четное число $2 n$ при любом натуральном $n \geqslant 2$. Значит, получается бесконечная серия примеров кусочно линейныхх изометрических отображений тора постоянной нулевой кривизны в $\mathbb{R}^{3}$. На рис. $7, a$ поверхность из этой серии имеет наименьший порядок оси симметрии, он равен $2 n=4$. 
В рассматриваемых примерах окрестность вершины поверхности с циклической последовательностью плоских углов $(\alpha, \alpha, \pi-\alpha, \pi-\alpha)$ имеет тип 0. Напротив, окрестность вершины поверхности с циклической последовательностью плоских углов $(\alpha, \pi-\alpha, \alpha, \pi-\alpha)$ представляет собой зонтик Уитни (конус над восьмеркой); это не вложение окрестности. Следует отметить, что при любом вложении этой окрестности вершина станет декоративной, так как при вложении в $\mathbb{R}^{3}$ тип этой окрестности может быть только одним из двух: либо ${ }^{11} 2$, либо ${ }^{12} 1$. В частности, отсюда можно заключить, что тор с указанными вьше развертками нельзя вложить или погрузить в трехмерное евклидово пространство $\mathbb{R}^{3}$.

Данные кусочно линейные изометрические отображения абстрактных замкнутых двумерных поверхностей постоянной нулевой кривизны в трехмерное евклидово пространство $\mathbb{R}^{3}$ не являются ни вложениями, ни погружениями. Однако ограничения этих отображений на поверхностях, проколотых в конечном числе точек ${ }^{13}$, становятся погружениями.

В заключение автор выражает глубокую благодарность рецензенту данной работы за ценные критические замечания.

\section{Список литературы}

1. Рурк К., Сандерсон.Б. Введение в кусочно линейную топологию. М.: Мир, 1974.

2. Александров А. Д. Выпуклые многогранники. М.-Л.: Гостехиздат, 1950.

3. Математическая энциклопедия. Т. 3. М.: Изд-во "Советская энциклопедия", 1982.

4. Харари Ф. Теория графов. М.: Мир, 1973.

5. Штогрин М. И. Положительность кривизны и выпуклость граней // Международная конференция, посвященная столетию Л.В. Келдыш. Москва, 24-28 августа 2004 г. Тез. докл.: Дискретная геометрия. М.: МИАН, 2004. С. 39-40.

6. Оловянишников С. П. Обобщение теоремы Коши о выпуклых многогранниках // Матем. сб. 1946. Т. 18. № 3. С. 441-445.

7. Cauchy A. Sur les polygones et polyedres, Second Memoire // J. Ecole Polytechnique. 1813. V. 9. P. 87-98.

Математический институт

Поступило в редакцию им. В.А. Стеклова

\footnotetext{
${ }^{11} \mathrm{~B}$ данном случае окрестность типа 2 составлена из двух пар вертикальных углов $(\alpha, \alpha)$ и $(\pi-\alpha, \pi-\alpha)$. При этом как вершина, так и все четыре ребра являются декоративными.

${ }^{12}$ Оба полукруга окрестности типа 1 составлены из двух секторов с углами $\alpha$ и $\pi-\alpha$. Два декоративных ребра и вершина предшествующей окрестности объединились в настоящее ребро.

${ }^{13}$ Из каждой абстрактной поверхности удаляются прообразы вершин зонтиков Уитни.
} 